\title{
Clinical significance of serum laminin and type-IV collagen levels in cutaneous melanoma patients
}

\author{
FARUK TAS, ELIF BILGIN, SENEM KARABULUT and DERYA DURANYILDIZ \\ Department of Medical Oncology, Institute of Oncology, University of Istanbul, Istanbul 34390, Turkey
}

Received September 25, 2015; Accepted April 19, 2016

DOI: $10.3892 / \mathrm{mco} .2016 .873$

\begin{abstract}
Laminin and type-IV collagen constitute a significant portion of the extracellular matrix. The objective of the present study was to evaluate whether the serum concentrations of laminin and type-IV collagen may serve as biomarkers for cutaneous melanoma. Sixty pathologically confirmed melanoma patients were enrolled in the study. Serum laminin and type-IV collagen levels were assessed using an ELISA. Thirty healthy controls were also examined. No significant differences in the baseline serum levels of laminin were identified between melanoma patients and healthy controls $(\mathrm{P}=0.45)$. However, the baseline serum levels of type-IV collagen were significantly elevated in melanoma patients compared with those in the control group $(\mathrm{P}<0.001)$. Clinical parameters, including patient age, gender, localization of lesion, histopathology, stage of disease, serum lactate dehydrogenase concentrations and responsiveness to chemotherapy were found not to be associated with the serum levels of laminin and type-IV collagen ( $\mathrm{P}>0.05)$. Furthermore, the serum levels of laminin and type-IV collagen had no prognostic value regarding the outcome for melanoma patients $(\mathrm{P}=0.36$ and $\mathrm{P}=0.26$, respectively). While laminin levels showed no diagnostic value, the serum concentrations of type-IV collagen were indicated to serve as a diagnostic marker in patients with cutaneous melanoma. In conclusion, type-IV collagen levels may be used as a diagnostic marker for cutaneous melanoma, while being void of any prognostic value.
\end{abstract}

\section{Introduction}

Melanoma is a malignant tumor type which is highly invasive and of metastatic character. These features depend on alterations in the extracellular matrix (ECM) to modify cell adhesion, migration and invasion.

The proteins laminin and type-IV collagen are major structural constituents of the basement membrane, and serve

Correspondence to: Dr Faruk Tas, Department of Medical Oncology, Institute of Oncology, University of Istanbul, no. 118 Millet Cad, Fatih, Capa, Istanbul 34390, Turkey

E-mail: faruktas2002@yahoo.com

Key words: serum, laminin, type-IV collagen, melanoma as promoters of cell proliferation, differentiation, adhesion and migration via integrins and other cell surface receptors (1-11). Increased expression of laminin and type-IV (1-7) collagen $(6,7,9-11)$ has also been demonstrated in melanoma cell lines with increased proliferation and metastatic potential (1-11).

While the function of the serum levels of laminin as biomarkers in patients with melanoma has been previously indicated (8), the majority of available data are from pre-clinical studies, and the concept remains to be further investigated and established in the clinical setting.

The present study investigated the concentration of circulating laminin and type-IV collagen in cutaneous melanoma patients and healthy controls and identified their association with patient prognosis, various clinical factors and responsiveness to chemotherapy in order to investigate whether their potential use as novel diagnostic or prognostic biomarkers.

\section{Materials and methods}

Patients. The cohort of the present study comprised 60 histologically confirmed cutaneous melanoma patients admitted to the Institute of Oncology (Istanbul University, Istanbul, Turkey). Patients with bi-dimensionally measurable disease without any history of chemotherapy or radiotherapy were included in the study. Staging was performed according to the staging system of the American Joint Committee on Cancer. The medical history of each patient was reviewed in detail; furthermore, physical examination and blood analyses were performed prior to the study. Patients with an Eastern Cooperative Oncology Group performance status of $\leq 2$ and suitable blood parameters received various standard immunotherapy or chemotherapy schemes, including interferon alpha, cisplatin, dacarbazine or temozolomide, as well as novel agents, including ipilimumab and vemurafenib based on the stage of the disease. The revised Response Evaluation Criteria in Solid Tumours version 1.1 were used for assessing the patients' responsiveness to chemotherapy.

For comparison, 30 age- and gender-matched healthy subjects were included in the study. Informed consent was obtained from all patients and the study was reviewed and approved by the local Ethics Committee.

Measurement of serum laminin and type-IV collagen levels. Serum samples were drawn from patients and healthy controls by venipuncture and clotted at room temperature on 
first admission prior to treatment. The sera were collected after centrifugation and immediately frozen at $-20^{\circ} \mathrm{C}$ until analysis.

Laminin ELISA (USCN Life Science Inc., Wuhan, China) and Collagen Type IV ELISA (USCN Life Science Inc.), double-antibody sandwich ELISA, were used for determination of the serum levels of laminin and type-IV collagen.

Statistical analysis. Values are expressed as median values and their ranges, standard deviation or standard error as indicated. Mann-Whitney U and Kruskal-Wallis tests were used to compare clinical and laboratory parameters to serum levels of laminin and type-IV collagen. The Kaplan-Meier test was used to estimate the survival and the differences in outcome were evaluated using the log-rank test. $\mathrm{P} \leq 0.05$ was considered to indicate a significant difference between values. SPSS 16.0 software (SPSS Inc., Chicago, IL, USA) was used for all statistical analyses.

\section{Results}

Patient characteristics. A total of 60 cutaneous melanoma patients were enrolled in this study. Of these, 13 were classified as stage I-II, 14 as stage III and 31 as stage IV, while the stage remained elusive for 2 patients (Table I). The median age of the patients was 53.5 years (range, 16-88 years). Patient characteristics and pathological features are listed in Table I.

Comparison of serum laminin and type-IV collagen between melanoma patients and healthy controls. There was no statistically significant difference in the baseline serum levels of laminin between cutaneous melanoma patients and healthy controls $(\mathrm{P}=0.45)$ (Table II). However, the baseline serum levels of type-IV collagen of the melanoma patients were significantly elevated compared with those in the control group $(\mathrm{P}<0.001)$ (Table II). Clinical parameters, including patient age, gender, localization of lesion, histopathology, stage of disease and serum lactate dehydrogenase (LDH) concentration were found to not be linked with the serum levels of laminin, type-IV collagen or responsiveness to chemotherapy $(\mathrm{P}>0.05)$ (Table III).

Survival analysis. The median survival time of the patients was 26.0 months [95\% confidence interval (CI): 21-30]. The one- and three-year overall survival rates were $76.3 \%$ (95\% CI: 64-88) and 51.0\% (95\% CI: 33-69), respectively. Survival was significantly decreased for patients with distant metastasis (M1) $(\mathrm{P}<0.001)$, advanced metastatic disease (M1c) $(\mathrm{P}=0.007)$, anemia $(\mathrm{P}=0.05)$, elevated erythrocyte sedimentation rate $(\mathrm{ESR})(\mathrm{P}<0.001)$, elevated serum $\mathrm{LDH}$ concentration $(\mathrm{P}<0.001)$ and no responsiveness to chemotherapy $(\mathrm{P}=0.01)$ (Table IV). However, serum laminin and type-IV collagen levels were not found to be of prognostic value in melanoma patients $(\mathrm{P}=0.36$ and $\mathrm{P}=0.26$, respectively) (Table IV and Figs. 1 and 2).

\section{Discussion}

Laminin and type-IV collagen have been shown to be present at the dermoepidermal junction and in the dermis
Table I. Patient characteristics and pathology.

Characteristics

Number of patients

Age, years

$<50 / \geq 50$

Gender

Male/female

Localization of lesion

Axial/extremity/unknown

$38 / 16 / 6$

Histopathology

Nodular/non-nodular/unknown

Stage of disease

I-II/III/IV/unknown

$13 / 14 / 31 / 2$

Tumor stage ${ }^{\mathrm{a}}$

T1-2/T3-4/unknown

$10 / 16 / 1$

Lymph node status $^{\mathrm{a}}$

Negative/positive

M1 stage

$\mathrm{M} 1 \mathrm{a}+\mathrm{b} / \mathrm{M} 1 \mathrm{c}$

Serum hemoglobin levels

Low/normal/unknown

$19 / 40 / 1$

Serum white blood cell count

Normal/elevated/unknown

$50 / 9 / 1$

Serum lactate dehydrogenase levels

Normal/elevated

$48 / 12$

Erythrocyte sedimentation rate

Normal/elevated/unknown

Responsiveness to chemotherapy ${ }^{\mathrm{b}}$

Yes/no/unknown

$10 / 11 / 10$

${ }^{a}$ In non-metastatic patients; ${ }^{b}$ in metastatic patients according to the revised Response Evaluation Criteria in Solid Tumours version 1.1.

neighboring benign melanocytic nevi, dysplastic nevi, and in melanoma (7). While a consistent loss of laminin and type-IV collagen from the basement membrane zone has been observed in areas adjacent to junctional melanocytic proliferation, this decrease cannot sufficiently distinguish between benign junctional proliferation and invasive early melanoma. The type of staining in the surrounding area of deeper invasive cells and intradermal nevus cells was similar and suggested that melanocytic cells at these sites may actually synthesize laminin and type-IV collagen (7).

Laminin, a major structural component of the basement membrane, drives cell proliferation, differentiation, adhesion and migration via integrins and other cell surface receptors (1-8). Increased laminin expression was also observed in melanoma cell lines and led to increased melanoma cell proliferation and metastatic potential (1-7). Similar to laminin, type-IV collagen is also an important structural component of the ECM and promotes 
Table II. Serum concentrations of laminin and type-IV collagen in melanoma patients and healthy controls determined by ELISA.

\begin{tabular}{lcccccc}
\hline & \multicolumn{2}{c}{ Patients $(\mathrm{n}=60)$} & & \multicolumn{2}{c}{ Controls $(\mathrm{n}=30)$} & \\
\cline { 2 - 3 } Parameters & Median & Range & & Median & Range & P-value \\
\hline Laminin $(\mathrm{pg} / \mathrm{ml})$ & 920.5 & $390.0-3,600.0$ & & 778.5 & $221.0-1,560.0$ & 0.45 \\
Type-IV collagen $(\mathrm{ng} / \mathrm{ml})$ & 54.5 & $27.0-123.0$ & & 10.5 & $3.0-20.0$ & $<0.001$ \\
\hline
\end{tabular}

melanoma cell adhesion, migration and invasion (6,7,9-11). It has been proposed that type-IV collagen expression ensures the development of scaffolding required for angiogenesis in melanoma (6,7,9-11). Type-IV collagen is a ECM protein and is highly common within the basement lamina and intima underlining the endothelium, excited chemotaxis of a number of human melanoma cell lines by engaging $\alpha 2 \beta 1$ integrin in vitro. Certain signal transduction incidents are driven by type-IV collagen through $\beta 1$ integrins, suggesting a significant role for NF- $\kappa \mathrm{B}$ in arranging chemotaxis of melanoma (9).

The majority of the findings on the abovementioned ECM proteins have been obtained from pre-clinical tissue-cell investigations (1-7,9-11). Apart from the present study, laminin and type-VI collagen have been assessed in the sera of melanoma patients in only one other study by Burchardt et al (8), who examined the serum levels of laminin, type-VI collagen, hyaluronan and tenascin- $\mathrm{C}$ in the sera of 6 patients with stage-I/II cutaneous melanoma and 6 patients with metastatic disease via ELISA. In addition, four melanoma cell lines were also analyzed for the expression of these markers. No differences in laminin serum concentrations were observed between healthy individuals and stage I/II melanoma patients or between stage I/II melanoma patients and those with metastatic disease. However, compared to those in healthy controls, serum laminin levels were significantly elevated in metastatic patients $(\mathrm{P}=0.018)$. Furthermore, the serum levels of type-VI collagen in stage I/II $(\mathrm{P}=0.004)$ and IV melanoma patients $(\mathrm{P}=0.007)$ were elevated compared to those in healthy controls. No significant difference was detected between stage I/II and IV patients. The authors suggested that these ECM markers were involved in melanoma growth and extracellular matrix remodeling in the course of melanoma progression (8). These findings indicated that collagen type-VI and laminin may serve as novel serum markers for melanoma progression.

In the present study, a total of 60 patients various clinical stages of melanoma were enrolled. Similar to the study by Burchardt et al (8), the serum concentrations of these markers were quantitatively analyzed by solid-phase ELISA. In the present study, the baseline serum levels of type-IV collagen were significantly elevated in melanoma patients compared to those in the control group $(\mathrm{P}<0.001)$. However, no statistically significant difference in the baseline serum levels of laminin were detected between melanoma patients and healthy controls. Furthermore, clinical parameters, including patient age, gender, localization of lesion, histopathology, clinical stage of disease, serum LDH concentration and responsiveness to chemotherapy were

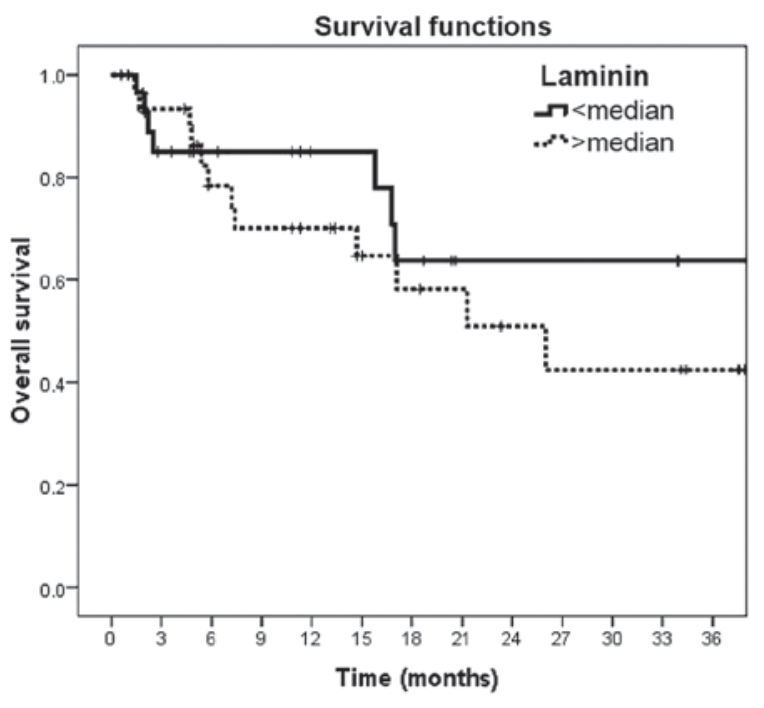

Figure 1. Survival curves in cutaneous melanoma patients in association with serum laminin levels $(\mathrm{P}=0.36)$.

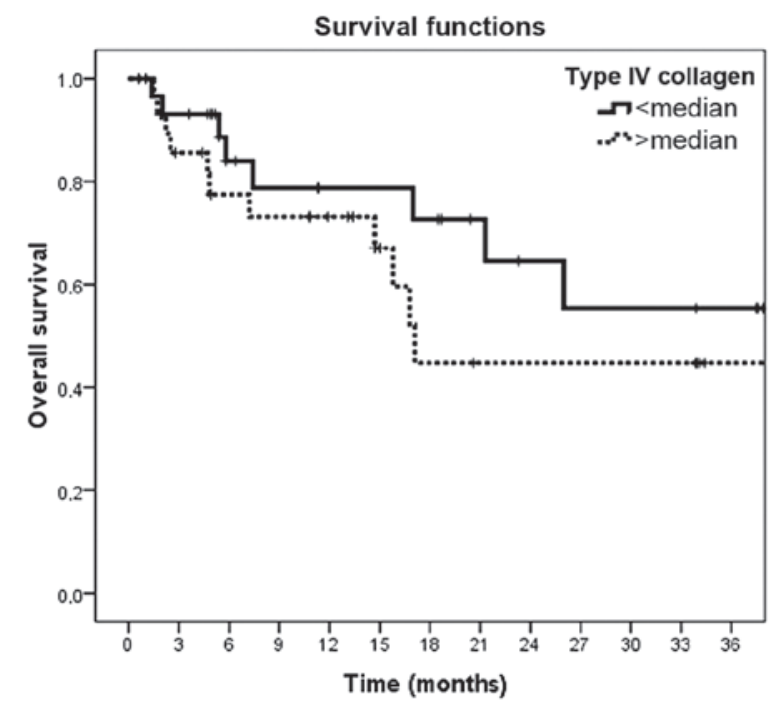

Figure 2. Survival curves in cutaneous melanoma patients in association with serum type-IV collagen levels $(\mathrm{P}=0.24)$.

found not to be associated with the serum levels of laminin and type-IV collagen. In addition, the serum levels of the two ECM proteins were shown to not be associated with patient survival and to therefore not to possess any prognostic value for melanoma patients.

In conclusion, the present study indicated that the serum levels of type-IV collagen may serve as a diagnostic 
Table III. Association of laminin and type-IV collagen levels with patient characteristics.

\begin{tabular}{|c|c|c|c|c|}
\hline \multirow[b]{2}{*}{ Characteristics } & \multicolumn{2}{|c|}{ Laminin } & \multicolumn{2}{|c|}{ Type-IV collagen } \\
\hline & Median (range) & P-value & Median (range) & P-value \\
\hline Age, years & & 0.73 & & 0.45 \\
\hline$\geq 50$ & $1008.0(221-3,530)$ & & $54.0(13-123)$ & \\
\hline$<50$ & $870.0(470-3,600)$ & & $54.5(28-102)$ & \\
\hline Gender & & 0.17 & & 0.98 \\
\hline Male & $921.0(390-3,600)$ & & $54.0(28-123)$ & \\
\hline Female & $860.0(409-3,530)$ & & $55.0(27-115)$ & \\
\hline Localization of lesion & & 0.87 & & 0.35 \\
\hline Axial & $919.0(390-3,600)$ & & $54.0(27-123)$ & \\
\hline Extremity & $891.0(409-3,530)$ & & $56.5(35-86)$ & \\
\hline Histopathology & & 0.32 & & 0.32 \\
\hline Non-nodular & $843.0(390-3,530)$ & & $54.0(28-102)$ & \\
\hline Nodular & $1008.0(521-3,600)$ & & $64.0(44-85)$ & \\
\hline Stage of disease & & 0.96 & & 0.60 \\
\hline I-II & $1010.0(390-1,557)$ & & $54.0(28-90)$ & \\
\hline III & $881.0(409-2,650)$ & & $54.0(36-86)$ & \\
\hline IV & $920.0(470-3,600)$ & & $58.0(27-123)$ & \\
\hline Tumor stage & & 0.09 & & 0.34 \\
\hline $\mathrm{T} 1-2$ & $776.5(390-1,375)$ & & $53.5(28-90)$ & \\
\hline T3-4 & $1054.0(409-2,650)$ & & $54.5(36-86)$ & \\
\hline Lymph node status (in M0 disease) & & 0.46 & & 0.71 \\
\hline Negative & $1061.0(390-1,557)$ & & $54.5(28-90)$ & \\
\hline Positive & $881.0(409-2,650)$ & & $54.0(36-86)$ & \\
\hline Metastasis status (in all patients) & & 0.87 & & 0.33 \\
\hline 0 & $921.0(390-2,650)$ & & $54.0(28-90)$ & \\
\hline 1 & $920.0(470-3,600)$ & & $58.0(27-123)$ & \\
\hline M1 stage & & 0.49 & & 0.13 \\
\hline$a-b$ & $960.0(470-3,530)$ & & $45.0(30-80)$ & \\
\hline $\mathrm{c}$ & $812.0(521-3,600)$ & & $62.0(27-123)$ & \\
\hline Serum hemoglobin levels & & 0.62 & & 0.23 \\
\hline Normal & $918.5(390-3,600)$ & & $53.5(28-100)$ & \\
\hline Low & $980.0(560-3,530)$ & & $60.0(35-123)$ & \\
\hline Serum white blood cell count & & 0.45 & & 0.87 \\
\hline Normal & $921.0(390-3,530)$ & & $54.5(28-115)$ & \\
\hline Elevated & $816.0(409-3,600)$ & & $56.0(28-123)$ & \\
\hline Erythrocyte sedimentation rate & & 0.63 & & 0.83 \\
\hline Normal & $911.5(390-2,690)$ & & $54.0(36-90)$ & \\
\hline Elevated & $964.5(470-3,530)$ & & $55.0(27-123)$ & \\
\hline Serum lactate dehydrogenase level & & 0.41 & & 0.93 \\
\hline Normal & $918.5(390-3,600)$ & & $54.5(28-102)$ & \\
\hline Elevated & $1090.0(521-3,530)$ & & $52.0(27-123)$ & \\
\hline Chemotherapy responsiveness $^{\mathrm{a}}$ & & 0.47 & & 0.09 \\
\hline Yes & $1005.0(562-3,600)$ & & $62.5(36-102)$ & \\
\hline No & $780.0(470-1,896)$ & & $45.0(27-100)$ & \\
\hline
\end{tabular}

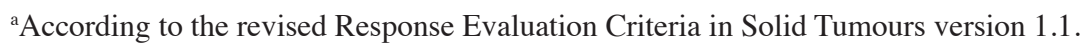


Table IV. Univariate analysis of survival.

\begin{tabular}{|c|c|c|c|}
\hline Characteristics & Median survival time $( \pm \mathrm{SE})$ (months) & Two-year survival rate $( \pm \mathrm{SD})(\%)$ & P-value \\
\hline Age, years & & & 0.20 \\
\hline$<50$ & $27.3(3.2)$ & $60.2(12.2)$ & \\
\hline$\geq 50$ & $24.4(3.3)$ & $53.1(11.6)$ & \\
\hline Gender & & & 0.35 \\
\hline Male & $25.1(3.1)$ & $58.5(10.1)$ & \\
\hline Female & $25.7(3.5)$ & $52.3(14.0)$ & \\
\hline Localization of lesion & & & 0.35 \\
\hline Axial & $27.1(2.8)$ & $61.7(9.7)$ & \\
\hline Extremity & $25.9(4.9)$ & $57.4(17.1)$ & \\
\hline Histopathology & & & 0.50 \\
\hline Non-nodular & $29.6(2.8)$ & $70.4(9.7)$ & \\
\hline Nodular & $23.0(4.3)$ & $58.3(25.1)$ & \\
\hline Tumor stage & & & 0.61 \\
\hline $\mathrm{T} 1-2$ & $35.6(2.3)$ & $88.9(10.5)$ & \\
\hline $\mathrm{T} 3-4$ & $34.3(3.4)$ & $66.7(27.2)$ & \\
\hline Lymph node status & & & 0.48 \\
\hline Negative & NR & $100.0(0)$ & \\
\hline Positive & NR & $90.9(8.7)$ & \\
\hline Distant metastasis & & & $<0.001$ \\
\hline No & $36.8(1.7)$ & $93.8(6.1)$ & \\
\hline Yes & $12.0(2.3)$ & $9.9(8.5)$ & \\
\hline M1 stage & & & 0.007 \\
\hline $\mathrm{a}-\mathrm{b}$ & $18.3(4.2)$ & $31.7(18.0)$ & \\
\hline $\mathrm{c}$ & $7.7(2.1)$ & NR & \\
\hline Serum hemoglobin levels & & & 0.05 \\
\hline Normal & $29.4(2.6)$ & $71.7(8.4)$ & \\
\hline Low & $17.9(3.3)$ & $24.0(14.4)$ & \\
\hline Serum white blood cell count & & & 0.59 \\
\hline Normal & $26.4(2.5)$ & $57.0(9.0)$ & \\
\hline Elevated & $14.5(2.5)$ & NR & \\
\hline Erythrocyte sedimentation rate & & & $<0.001$ \\
\hline Normal & $30.9(2.5)$ & $71.1(11.2)$ & \\
\hline Elevated & $14.1(3.7)$ & $21.4(12.5)$ & \\
\hline Serum lactate dehydrogenase levels & & & $<0.001$ \\
\hline Normal & $30.0(2.3)$ & $66.4(9.3)$ & \\
\hline Elevated & $4.6(0.8)$ & NR & \\
\hline Chemotherapy responsiveness $^{\mathrm{a}}$ & & & 0.01 \\
\hline Yes & $17.6(3.9)$ & $27.4(16.2)$ & \\
\hline No & $4.5(0.7)$ & NR & \\
\hline Laminin & & & 0.36 \\
\hline Low (<median) & $28.9(3.3)$ & $63.7(11.8)$ & \\
\hline High (>median) & $23.2(3.0)$ & $50.9(11.6)$ & \\
\hline Type-IV collagen & & & 0.24 \\
\hline Low (<median) & $27.4(3.1)$ & $64.6(11.6)$ & \\
\hline High (>median) & $23.1(3.5)$ & $44.7(12.4)$ & \\
\hline
\end{tabular}

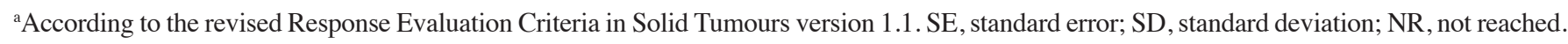


biomarker in melanoma patients, while laminin levels were of no diagnostic value. However, the serum levels of neither of the two ECM proteins had any predictive or prognostic value in melanoma patients. The small size of the patient cohort and the short time to follow-up of the present study (36 months) represent significant limitations and may have affected the results. However, the present study contributed to the current understanding of the roles of these ECM proteins in the pathology of cutaneous melanoma in an anatolian or Asia minor population, and patients at various stages were included. Studies on larger patient populations are required to further determine the exact role of these biomarkers in cutaneous melanoma patients.

\section{References}

1. Chen HB, Chen L, Zhung JK, Chow VW, Wu BQ, Wang ZH, Cheng SB and Chew EC: Expression of laminin in metastatic melanoma cell lines with different metastatic potential. Anticancer Res 21: 505-508, 2001.

2. Mortarini R, Gismondi A, Maggioni A, Santoni A, Herlyn M and Anichini A: Mitogenic activity of laminin on human melanoma and melanocytes: Different signal requirements and role of beta 1 integrins. Cancer Res 55: 4702-4710, 1995.

3. Oikawa Y, Hansson J, Sasaki T, Rousselle P, Domogatskaya A, Rodin S, Tryggvason K and Patarroyo M: Melanoma cells produce multiple isoforms and strongly migrate on $\alpha 5$ laminin(s) via several integrin receptors. Exp Cell Res 317: $1119-1133,2011$.
4. Lugassy C, Torres-Muñoz JE, Kleinman HK, Ghanem G, Vernon S and Barnhill RL: Overexpression of malignancy-associated laminins and laminin receptors by angiotropic human melanoma cells in a chick chorioallontoic membrane model. J Cutan Pathol 36: 1237-1243, 2009.

5. Gradilone A, Gazzaniga P, Cigna E, et al: Fibronectin and laminin expression in sentinel lymph nodes of patients with malignant melanoma. Br J Dermatol 157: 398-401, 2007.

6. Ramos DM, Berston ED and Kramer RH: Analysis of integrin receptors for laminin and type IV collagen on metastatic B16 melanoma cells. Cancer Res 50: 728-734, 1990.

7. Mackie RM, Clelland DB and Skerrow CJ: Type IV collagen and laminin staining patterns in benign and malignant cutaneous lesions. J Clin Pathol 42: 1173-1177, 1989.

8. Burchardt ER, Hein R and Bosserhoff AK: Laminin, hyaluronan, tenascin-C and type VI collagen levels in sera from patients with malignant melanoma. Clin Exp Dermatol 28: 515-520, 2003.

9. Hodgson L,Henderson AJ and Dong C: Melanoma cell migration to type IV collagen requires activation of NF-kappaB. Oncogene 22: 98-108, 2003.

10. Pasco S, Brassart B, Ramont L, et al: Control of melanoma cell invasion by type IV collagen. Cancer Detect Prev 29: 260-266, 2005.

11. Daniels KJ, Boldt HC, Martin JA, et al: Expression of type IV collagen in uveal melanoma: Its role in pattern formation and tumor progression. Lab Invest 75: 55-66, 1996. 